\title{
Comparison of fixed and living biological cells parameters investigated with a digital holographic microscope
}

\author{
P. Nienałtowski*, M. Baczewska, M. Kujawińska \\ Faculty of Mechatronics, Warsaw University of Technology, Sw. A. Boboli 8, 02-525 Warszawa,
}

Received December 30, 2020; accepted March 28, 2020; published March 31, 2020

\begin{abstract}
Statistical analysis and comparison of biophysical parameters of living and fixed, mouse embryonic fibroblasts cells are presented. Parameters calculations are based on phase measurements performed by means of a digital, holographic microscope. The phases are retrieved from off-axis, image plane holograms, followed by custom image segmentation and statistical analysis of cells' surface, phase volume and dry mass. The results indicated statistically significant differences between fixed and living cell parameters, which is an important message for setting the methodology for further diagnosis based on quantitative phase (label-free) analysis.
\end{abstract}

Currently, microscopic studies of cell cultures and histopathology samples slowly convert from imaging of chemically stained or labeled microsamples to label-free quantitative phase analysis of transparent microobjects. One of the most popular methods suitable for such analysis is digital holographic microscopy (DHM) [1]. DHM enables measuring many parameters of biological samples such as integrated optical path difference (integrated phase), dry mass, shape and in some cases cell volume. Currently, a lot of studies are carried out on fixed cells, which are easier to handle than living cells. However, many problems require experiments with living cells. The data from both investigations are often mixed and clear interpretation of the results is difficult if not impossible. Most of the previous studies have devoted little attention to the analysis of the impact of fixation process on the values of cell parameters. This work aims to compare the parameters of fixed and living biological cells and answer the question whether the results of their measurements can be treated equally and used for machine learning purposes and further diagnosis [2].

The measurements described in this article were carried out at a multimodal system combining a white light optical microscope and a digital holographic microscope (Fig. 1). DHM works in the Mach-Zehnder interferometer configuration and in-plane, off-axis hologram capture as described in detail in [3].

*E-mail: patryknienaltowski@gmail.com
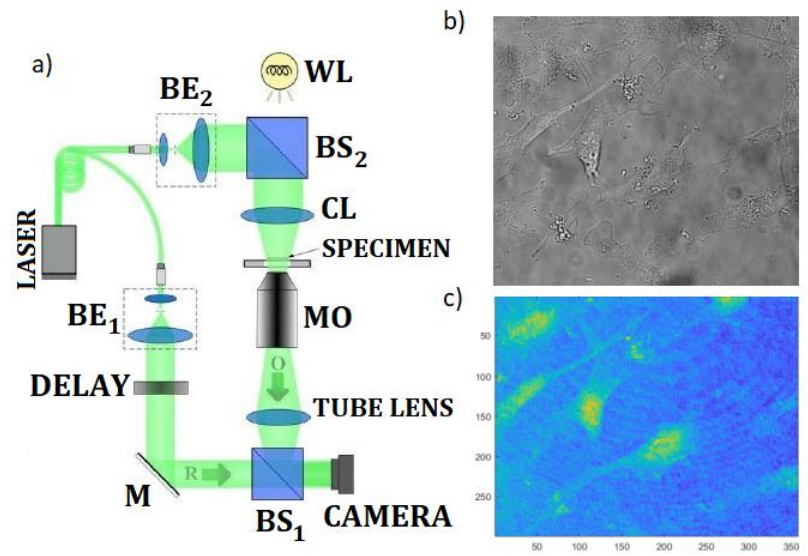

Fig. 1. a) The scheme of the DHM setup [2], b) a white light image and c) a phase map of example NIH3T3 cell culture. WL, white light source; BE1/BE2, beam expanders; BS1/BS2, nonpolarizing beam splitters; CL, condenser lens; MO, microscope objective; M, mirror.

Mouse embryonic fibroblasts cells from NIH3T3 line were chosen for the research, as an example of the basic type of cells. The conditions for the preparation of cell cultures tested in the laboratory of the Westphalian Wilhelm University in Münster were compatible with the international standard [4]. Several holograms have been captured from different areas of the cell culture in order to provide a sufficient number of cells for further statistical analysis. In this work, we have focused on investigations of the influence of the most popular fixation process paraformaldehyde fixation (PFA) - on biophysical parameters of the cells.

The phase $\bmod (2 \pi)$ maps are retrieved from holograms using the Fourier Transform Method. After phase unwrapping, the phase maps have to be postprocessed in order to enable correct phase images segmentation and determination of biophysical parameters of individual cells. Phase postprocessing includes two steps:

- removal of a residual linear term from the phase map, which is realized by subtracting the best-fitting plane. It allows to obtain background stability, which will contribute to better cell segmentation;

- removal of systematic errors in the recovered phase resulting from the aberration of DHM optical system including imperfections of a Petri dish. This step is simple 
if a reference phase map can be captured (for each measurement) and subtracted from the actual phase measurement. However, in this case, it is impossible and therefore the method of averaging measurements from one series has been applied [5].

The conditions for using this method are: (i) the presence of a specimen with a large dimension in comparison with the unitary field of view and (ii) stochastic distribution of the component objects (cells). They ensure that information about objects will not be removed in the averaging process. Then average subtraction is applied to each of the processed holograms separately. The integrated phase map in this processing path is determined as:

$$
\varphi(x, y)=\varphi_{z}(x, y)-\bar{\varphi}(x, y),
$$

where $\varphi_{z}-$ the measured phase, $\bar{\varphi}-$ the average phase.

The postprocessing algorithm enables to achieve background stability, emphasize the measured objects and eliminate errors that may affect the calculated parameters and the results of further analysis. This is a key step to make reliable measurements.

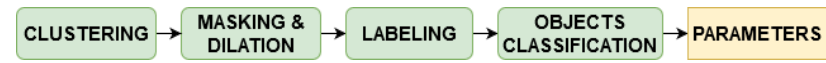

Fig. 2. Block diagram of the image segmentation process.

Based on efficiency, simplicity, and usability for biological data processing, two segmentation algorithms were selected for testing - the watershed method [6] and the clustering method [7]. As an input image, a corrected phase map from a digital holographic microscope was used. The results of the segmentation by means of both algorithms are shown in Fig. 3.
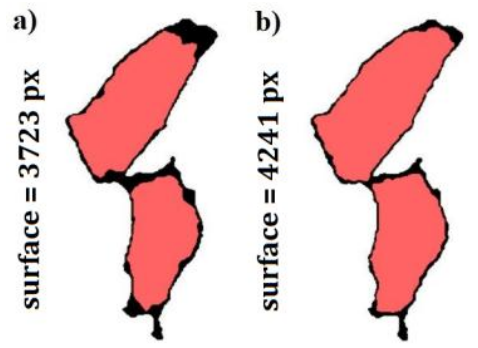

Fig. 3. Segmentation results and calculated cell surfaces using the method of: a) watershed segmentation and b) clustering segmentation with the k-means algorithm; the area marked in red has been classified as a cell, the black area is the original biological cell.

The final selection of the segmentation algorithm was based on a visual comparison of the effects of segmentation with both methods, the main criterion was the degree of coverage of the segmented cell with a reference. For further processing of a phase, the k-means method of clustering was selected. It is a non-hierarchical open-source algorithm implemented in Matlab [7]. The schemes of phase maps processing are shown in Fig. 4. After division into clusters, the cluster containing background is removed from the image. The detection of a cluster containing background is based on a number of pixels. Due to the nature of the measurements, the background cluster contains the majority of pixels. After choosing clusters containing cells, the process of creating a binary mask follows. Areas in the image containing cells have a value of 1 and the background has a value of 0 . The next step is to perform a few morphological operations such as dilatation with a spherical structural element [8]. The prepared binary image is labeled to analyze each cell separately. In this algorithm, an 8-pixel connection condition was used. The last stage of image segmentation is based on surface classification of objects to eliminate connected objects that are not cells (Fig. 4b).

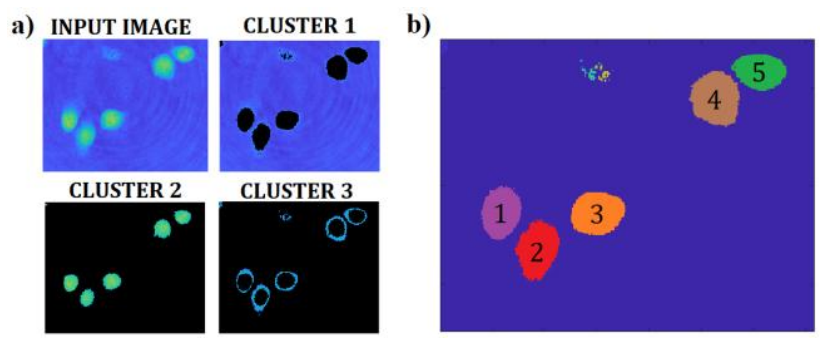

Fig. 4. The schemes of phase processing: a) clustering of the image; cluster 1 contains the background, cluster 2 contains the nucleus of the cell, cluster 3 contains the plasmatic structure, b) separation of individual cells from phase maps.

Determined cell parameters were based on phase values from which single cells were segmented. The parameters on which the statistical analysis is based are:

(i) cell surface $S$, which is calculated using dimensions of the pixel $a^{2}$ in sample plane and number $N$ of pixels classified as a cell:

$$
S=N \cdot a^{2},
$$

(ii) cell phase volume $V_{\varphi}$ (OVD - optical volume difference) which is not the actual volume of the cell, but the equivalent of the cell volume, based directly on the optical path difference $O P D$ :

$$
V_{\varphi}=\frac{\lambda}{2 \pi} \cdot \overline{O P D} \cdot S
$$

(iii) dry mass $M$, which quantifies the mass of nonaqueous cell material:

$$
M=\frac{1}{\alpha} \frac{\lambda}{2 \pi} \cdot \overline{O P D} \cdot S,
$$

where $\alpha-$ is the proportionality constant called the specific refractive increment. 
As shown above, the phase values of cells (OPD) are closely related to the biophysical parameters, namely the cell phase volume (OVD) and cell dry mass (M).

Two-sample t-Tests were carried out to thoroughly analyze the parameters. For dry mass and OVD, the same null hypothesis $H_{0}: \mu_{1}=\mu_{2}$ with the equality of two means and the alternative hypothesis $H_{1}: \mu_{1}<\mu_{2}$ (average value for fixed cells is smaller) were formulated. For cell surface measurements, the null hypothesis $H_{0}: \mu_{1}=\mu_{2}$ was formulated with the equality of two means and the alternative hypothesis $H_{1}: \mu_{1} \neq \mu_{2}$.

For analysis, 25 fixed cells and 20 living cells were measured in the laboratory of the Westphalian University of Wilhelm in Münster under the same measurement conditions and with identical microscope settings.

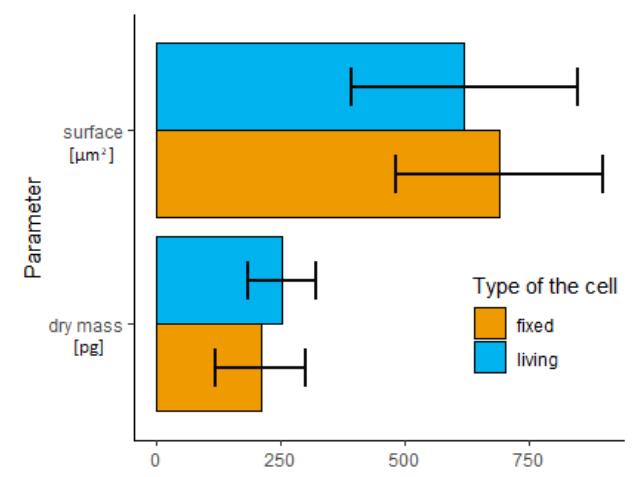

Fig. 5. Comparison of average dry mass and surface values for fixed and living cells with standard deviations.

Figure 5 presents the results of statistical analysis of cell parameters: average dry mass and surface values of fixed and living cells. The t-Test result for dry mass measurements rejected the null hypothesis (p-value 0.0397 ) by accepting an alternative hypothesis. The results of OVD measurements, being a parameter proportional to the dry mass, are as follows: for the fixed cells the average was $45.10 \mu \mathrm{m}^{3}$ with $\mathrm{SD}=12.88 \mu \mathrm{m}^{3}$ and for the fixed cells the average was $39.88 \mu \mathrm{m}^{3}$ with $\mathrm{SD}=$ $17.11 \mu^{3}$. For OVD values, the hypothesis test also rejected the null hypothesis ( $\mathrm{p}$-value 0.0397 ). Test results for surface measurements did not reject the null hypothesis of mean equality ( $p$-value 0.2981 ). The values of the coefficient of variation indicate that in the case of surface measurements there is less variation than in the measurement of parameters proportional to the integrated phase. The large standard deviation values determined for all parameters are the result of calculating the parameters of cells from different areas of the specimen, natural diversity of cells and a relatively small number of cells in the statistical probe.
The goal of the comparison of living and fixed cells was to examine if integrated phase measurements and related biophysical parameters of both cell types can be equally applied for future applications in digital phase histopathology and/or remote diagnostic supported by deep learning procedures [2]. The tests performed show that there are statistically significant differences in dry mass (and OVD) between fixed and living cells. Fixed cells have a smaller dry mass than living cells. Also, by measuring the cell surface, it was checked whether the difference in the dry mass is due to the changes in the size of the cells. The statistical analysis indicated that the changes in dry mass are caused by the PFA fixation process. It means that PFA fixation significantly affects the biological parameters of the cells, which has to be taken into account while applying this data for further quantitative analysis leading to meaningful diagnosis. The reported results are based on measurement of one specific cell line and show changes in the global (for whole cell) biophysical parameters (M, OVD, S). It is urgently needed to perform similar investigation for different cell lines, their larger statistical representation and analysis of changes in subcellular structures. However, the aim of the present work was to bring, as early as possible, attention to the differences between fixed and living cells so that phase based data originated from both cell types are not mixed and simultaneously entered into the AI databases and used for diagnostic purposes.

The research leading to the described results was carried out within the program TEAM TECH/2016-1/4 of the Foundation for Polish Science, co-financed by the European Union under the European Regional Development Fund. We thank the research team from Westphalian Wilhelm University in Münster for providing measuring devices and cell cultures for these studies.

\section{References}

[1] K. Alm et al., InTech, doi: 10.5772/54505, 2013.

[2] Y. Rivenson, Y. Wu, A. Ozcan, Light: Science Appl. 8, 85 (2019)

[3] J. Min et al., Opt. Lett. 42(2), 227 (2017).

[4] M. Baczewska, Measurements and analysis of cells and histological skin sections based on digital holographic microscopy (WUT Master Thesis, 2018).

[5] P. Stępień, D. Korbuszewski, M. Kujawińska, ETRI J. 41(1), 73 (2019).

[6] S. Beucher, The Watershed Transformation Applied to Image Segmentation, Scanning microscopy (Supplement 6, 2000).

[7] J.A. Hartigan, M.A. Wong, A K-Means Clustering Algorithm (Applied Statistics, 1979).

[8] J. Serra, Image Analysis and Mathematical Morphology (Academic Press, 1982).

[9] P. Girshovitz, N.T. Shaked, Biomed. Opt. Expr. 3(8), 1757 (2012). 